\title{
Are Footedness and Lateral Postures Better Predictors of Hemispheric Dominance Than Handedness: A Cross-sectional Questionnaire-Based Clinical and Pedigree Study
}

\author{
Aparna Muraleedharan ${ }^{1}$ Saranya Ragavan ${ }^{1} \quad$ Rema Devi $^{1}$
}

${ }^{1}$ Department of Anatomy, Pondicherry Institute of Medical Sciences, Puducherry, India

J Neurosci Rural Pract 2020;11:130-134 Address for correspondence Aparna Muraleedharan, MD, Department
of Anatomy, Pondicherry Institute of Medical Sciences, Kalapet,
Puducherry-605014, India (e-mail: aparnamuraleedharan18@gmail.com).

\begin{abstract}
Keywords

- lateral postures

- sidedness

- hand clasping

- arm folding

- leg folding

- retraining

Objective Approximately 9 out of 10 individuals show right-hand dominance. The remaining $10 \%$ include left-hand dominant and mixed handed/ambidextrous individuals. Laterality, lateral postures or lateral preference is defined by various authors as the most comfortable posture that an individual prefers to assume. The current study aims at finding the distribution of these lateral postures and their correlation with handedness/dominant side.

Materials and Methods The study was done in medical and nursing students in the age group 18 to 22 years. The patients were asked to fill a self-assessment questionnaire to determine handedness and footedness. The laterality traits were observed by asking the patients to do by themselves after an untailored demonstration.

Statistical Analysis The relationship between study parameters was assessed using Chi-square test. Pearson's correlation test was done to assess the strength of association between comfort ratings of the various laterality postures.

Results In our study, $96.5 \%$ were right handed and $79.9 \%$ of patients were right footed. Also, 50.8\% preferred right-hand clasping, 53.9\% preferred left-arm folding, and $60.6 \%$ preferred right-leg folding. A statistically significant correlation was seen between foot preference and lateral postures and arm folding and hand clasping $(p<0.05)$. A significant association was also found between handedness and positive history of retraining. Sidedness did not follow any Mendelian pattern of inheritance. Conclusions Handedness is affected by genetic and environmental factors. Since cultural and traditional practices can affect the lateral postures and handedness, it is important to know the relationship between laterality postures and sidedness and the impact of retraining on them. These postures are measures of cerebral dominance and are clinically important.
\end{abstract}

\section{Introduction}

The term handedness means the preference with which a person uses a particular hand for trained activities that need special skill and coordination like writing, drawing, cutting, and eating. Approximately, $90 \%$ of individuals are righthanded either by birth or following retraining of left handedness. 8 to $10 \%$ of people are born left handed. Some are ambidextrous by birth or because children who exhibit left handedness are retrained by force due to cultural reasons to 
write and eat with their right hand which may have resulted in ambidexterity or incomplete handedness. There are two aspects of handedness, proficiency and preference. Proficiency can be measured by assessing muscle strength using grip strength and skillfulness by asking to perform fine motor tasks. Preference is referred to as the hand chosen by an individual to do an activity. This can be assessed by asking the patient to indicate the hand or foot chosen for a task by using a self-assessment questionnaire. ${ }^{1}$

Increasing bodies of evidence suggest that, though handedness is a better determinant of cerebral dominance, foot preference (FP) predicts cognitive and language construct better than hand preference., ${ }^{2,3}$ Footedness could be a better predictor of actual hemispheric dominance at least in cultures where retraining of handedness is still rampant. But most studies have not taken the footedness into account. Moreover, the existing data are conflicting. In the past, most of the questionnaires to assess sidedness had majority of the questions for assessing handedness (usually $>10$ ) and a very few questions for FP (usually <4).

Lateral posture is the comfort posture assumed by an individual in routine activities which need little or no skill or training. Hand clasping (HC), arm folding (AF), leg folding (LF), and FP are some traits of laterality. ${ }^{4}$ Mohr et al tried to correlate the lateral postures to handedness and found that the strength of right handedness was significantly higher in people who had left-hand clasping (LHC) than in people who had right-hand clasping (RHC). Left-arm folding (LAF) was more common arm folding posture among right-hand dominant individuals. ${ }^{5}$

In the past, educators and psychologists believed that left handedness indicated a personality problem in the child and hence advocated switching of handedness. Several researchers later proved that retraining of handedness eventually resulted not only in weak degree of laterality but also dangerous problems like stuttering and stammering. ${ }^{6}$ Some recent studies have also shown abnormal signal transmission from dominant to nondominant hemisphere in such people. ${ }^{7}$ The population in this part of the world has cultural and traditional practices that can highly affect the handedness and to a variable extent footedness but very rarely the lateral postures. There is paucity of literature regarding a similar study that incorporates all these laterality measures and sidedness. ${ }^{8,9}$ This study also incorporates history of retraining and switching of handedness.

Ninety-two percent of individuals are right handed. Literature shows that for over 5 millennia left-hand dominance is seen in around $8 \%$ of the population and this has almost been constant. Family studies of handedness have failed to provide a logical explanation to the occurrence of left handedness. There are environmental and genetic factors that predict handedness and so left handedness is multifactorial in inheritance. Some studies have attributed hypoxia during intrapartum period for the occurrence of left handedness in the foetus. Some studies have shown that cerebral dominance is determined partly by the foetal testosterone levels. Hence, this could also affect handedness.
The current study aims at finding the distribution of these lateral postures and their correlation with handedness/ dominant side. The study is done in medical and nursing undergraduates in the age group 18 to 22 years. Since cultural and traditional practices can affect the lateral postures and handedness, it is important to know the relationship between laterality postures and sidedness and the impact of retraining on them. These postures are measures of cerebral dominance and are probably clinically important in the diagnosis, treatment, and prognosis of strokes and aphasias, speech problems, and dyslexia. ${ }^{10}$

\section{Materials and Methods}

\section{Patients}

The study was done in MBBS and BSc nursing students in the age group 18 to 22 years. Those students with any physical disability or a limb deformity were the exclusion criteria. The institute research and ethics committee approval was obtained prior to the conduct of the study.

\section{Data Collection}

After obtaining written informed consent, each of the patients were asked to fill a self-assessment questionnaire to determine the handedness and footedness. The questionnaire included a set of 27 questions to assess sidedness (20 for handedness and 7 for footedness) which are practically similar to various other authorized questionnaires. ${ }^{11}$ As handedness in this country is influenced by several social, cultural, traditional factors, it is important to design a handedness inventory that takes into account the prevailing cultural factors. ${ }^{12}$ So, some questions are reasonably modified according to the Indian scenario and, hence, was tested for content validity. It also contained questions regarding personal information like history of any injury or retraining. The answers are scored from 2 to +2 (where $+2=$ always uses right, $+1=$ usually uses right, $0=$ equally uses right and left/ equivocal, -1 = usually uses left, and $-2=$ always uses left). This scoring system was used for assessing the strength of the hand/FP. The original questionnaire contained 32 items. This was tested for validity by three subject experts and, in some questions, changes were made to suit the cultural practices followed in our setting. The questionnaire was then tested on 20 patients to check the cultural relevance and applicability. Five items were removed from the original questionnaire due to confusing answers. Three questions were modified to suit the cultural and traditional practices. The final version therefore contained 27 items. This was again administered to the same participants in order to confirm accuracy of responses. The reliability was found to be high. The modified questionnaire with 27 questions was, thus, used for the study.

The genetic traits, such as $\mathrm{HC}, \mathrm{AF}$, and LF were observed by asking the patients to do by themselves after an untailored demonstration. There are four possible HC positions which are LHC with normal finger clasping and abnormal finger clasping ( $\mathrm{LHCl}$ ) and $\mathrm{RHC}$ with normal finger clasping and abnormal finger clasping (RHCI). There are two possible arm folding positions, right arm folding (RAF) where 
the right forearm is over the left and left arm folding (LAF) where the left forearm is over the right. The two possible LF positions are right leg folding (RLF) and left leg folding (LLF). ${ }^{4}$ The patients are first asked to assume their natural position for each lateral posture, HC, AF, and LF. This is taken as their lateral posture preference. Then they are asked to try out the other possible positions and their comfort rating is marked on a five-point Likert's scale. A short history of retraining was also included in this questionnaire by which the patients can elaborate on history of conversion from left handedness, why and how. It took approximately 15 to 20 minutes to answer the questionnaire and demonstrate the postures.

A three-generation pedigree analysis was then done in families of all 258 medical and nursing undergraduate students to assess the inheritance pattern of handedness. A pedigree chart was drawn for each student by taking a history using a questionnaire containing questions regarding handedness in siblings, parents, and grandparents.

\section{Statistical Analysis}

The relationship between handedness, FP, AF, HC, and LF was assessed using Chi-square test. Pearson's correlation test was done to assess the strength of association between comfort ratings of the various laterality postures.

\section{Results}

A total of 258 students participated in this study. Their mean age was $18.9 \pm 0.9$ years. Majority of the sample was females $(n=164 ; 63.6 \%), 96.5 \%(n=249)$ were right handed. $0.4 \%(n=1)$ was left handed, $3.1 \%(n=8)$ were ambidextrous, $12.02 \%(n=31)$ of patients gave a positive history of retraining of left handedness during childhood, $79.9 \%(n=203)$ were right footed, 7.5\% ( $n=19)$ were left footed, and $12.6 \%(n=32)$ were mixed footed; among them, 50.8\% $(n=131)$ preferred RHC, 53.9\% $(n=139)$ preferred LAF, and 60.5\% $(n=156)$ preferred RLF. Majority of subjects showed right dominance. The difference in the of distribution of handedness, FP, HC, AF and LF in males and females was not found to be statistically significant i.e. there was no gender differences in frequency of distribution of these variables.

Handedness and footedness showed a statistically significant correlation $\left(\chi^{2}: 14.01 ; p<0.05\right)$. The other laterality postures and handedness showed no significant association (-Table 1). Footedness was found to significantly correlate with AF and HC $(p<0.05)$. But LF and footedness showed no significant association (-Table 2). Majority of right footed patients had LAF and RHC and this was statistically significant.

The relationship between strength of preference of laterality postures, RHC, LHC, RAF, and LAF (-Table $\mathbf{3}$ ) was assessed using Chi-square test. Pearson's correlation test showed that the comfort levels of RHC and LAF was found to have a statistically significant association $(p<0.05)$. This study failed to demonstrate any statistically significant correlation between any other lateral postures with handedness or footedness.
Table 1 Comparison of handedness with other variables

\begin{tabular}{|l|l|l|}
\hline & $\chi^{2}$ & $p$-Value \\
\hline Handedness vs. HC & 4.147 & 0.84 \\
\hline Handedness vs. FP & 14.01 & $0.007^{\text {a }}$ \\
\hline Handedness vs. AF & 2.9 & 0.82 \\
\hline Handedness vs. LF & 14.3 & 0.07 \\
\hline
\end{tabular}

Abbreviations: AF, arm folding; FP, footedness/foot preference; HC, hand clasping; LF, leg folding.

${ }^{a} p$-Value $<0.05$.

Table 2 Comparison of footedness/foot preference (FP) with $\mathrm{HC}, \mathrm{AF}$, and $\mathrm{LF}$

\begin{tabular}{|l|l|l|}
\hline & $\chi^{2}$ & $p$-Value \\
\hline FP vs. HC & 15.03 & $0.04^{\mathrm{a}}$ \\
\hline FP vs. AF & 12.95 & $0.04^{\mathrm{a}}$ \\
\hline FP vs. LF & 3.8 & 0.87 \\
\hline
\end{tabular}

Abbreviations: AF, arm folding; FP, footedness/foot preference; HC, hand clasping; LF, leg folding.

${ }^{\mathrm{a} p}$-Value $<0.05$

Table 3 Comparison of strengths of preference between various laterality postures

\begin{tabular}{|l|l|l|}
\hline & $\chi^{2}$ & $p$-Value \\
\hline RHC vs. RAF & 11.65 & 0.77 \\
\hline RHC vs. LAF & 28.451 & $0.028^{\text {a }}$ \\
\hline LHC vs. RAF & 7.39 & 0.5 \\
\hline LHC vs. LAF & 1.097 & 0.19 \\
\hline
\end{tabular}

Abbreviations: LAF, left-arm folding; LHC, left-hand clasping; RAF, right-arm folding; RHC, right-hand clasping.

a $p$-Value $<0.05$.

Thirty-one patients gave a history of using left hand for routine activities in early childhood and had a positive history of retraining for switching of handedness. Among them, 28 had been converted to right handed, 2 of them were ambidextrous and 1 had continued to be left handed. The association between handedness and retraining was found to be statistically significant $\left(\chi^{2}: 8.803, p<0.05\right)$.

None of the family members of left handed students exhibited left handedness. Twenty-four right handed students had a close family member who was left handed. Two students had left handed father and paternal grandfather. Thus, there were 26 left handed relatives in all (-Table 4).

\section{Discussion}

Our study aimed at finding the distribution of handedness and lateral postures, estimate the comfort levels of various laterality preferences, and to find the association between handedness and these postures. As shown in previous literature, majority of patients in our study were right handed and right footed. Handedness and footedness showed a significant association ( - Table 1). Among the lateral postures, RHC, 
Table 4 Frequency of left handedness in family members

\begin{tabular}{|l|l|}
\hline Relative & No. left handed (26) \\
\hline Brother & 9 \\
\hline Sister & 6 \\
\hline Father & 4 \\
\hline Mother & 2 \\
\hline Maternal grandfather & 0 \\
\hline Maternal grandmother & 3 \\
\hline Paternal grandfather & 2 \\
\hline Paternal grandmother & 0 \\
\hline
\end{tabular}

LAF, and RLF were more common. This is in concordance with existing literature. The Likert scale comfort levels of RHC and LAF also had a statistically significant correlation ( - Table $\mathbf{3}$ ), that is, most of the patients who found RHC comfortable also found that LAF was comfortable, and this was more common among right-handed individuals. This was found to be the same among both the genders. Previous researchers have also found that majority of right handers clasp with their right thumb on top (RHC) and fold their arms with left forearm on top (LAF). But it has been reported that right hand dominant patients with these laterality postures have a weaker degree of handedness.

Some previous researchers have found significant associations between handedness and AF, and handedness and $\mathrm{HC}^{4,13}$ But in the current study, handedness did not show a statistically significant association with AF or HC (- Table 1). Familial studies on $\mathrm{HC}$ and $\mathrm{AF}$ have shown a genetic influence though their inheritance pattern does not show any specific Mendelian pattern of inheritance. ${ }^{14,15}$ The case was the same for LF as well. Literature has shown a higher rate of RLF (62\%) than $\operatorname{LLF}(26 \%)$ and around $12 \%$ have no preference or show an indifference to $\mathrm{LF}^{16}$ In our study, we found a similar incidence of RLF (60.5\%) but the incidence of LLF was only 9.7\%. A larger number of patients (31\%) showed no specific preference for LF, that is, they were equally comfortable with RLF and LLF $(26 \%)$ or showed an indifference $(4.7 \%)$ to LF. The most important reason for showing indifference was found to be obesity and female gender. Around 3.5\% of patients showed indifference to $\mathrm{AF}$ as well. The reasons reported by the patients were female gender, obesity, and religious practices. This study has thus explored the possible reasons for indifference to a lateral posture which has not been reported in previous studies.

The studies that relate FP to cerebral dominance is increasing. Several authors have studied the relation between FP and language centers in brain. ${ }^{17}$ Elias et al studied the relationship between FP and emotional lateralization. ${ }^{2}$ Several studies have shown significant correlation between lateral postures and cerebral dominance. ${ }^{18,19}$ Footedness and lateral postures of upper limb (AF and $\mathrm{HC}$ ) showed statistically significant correlation (-Table 2). Hence, we opine that footedness could be a better predictor of actual hemispheric dominance at least in cultures where retraining of handedness is still rampant. ${ }^{2,3}$ This has not been shown in several previous studies. ${ }^{3,13}$ The ability of footedness to determine hemispherical dominance than handedness could be due to the possible effects of retraining of left handedness that is commonly seen in our society much more than left footedness. Indian parents do train their children to begin an activity with their right foot forwards like entering a holy place, a new house or an auspicious ceremony; but many of the day-to-day activities with the foot are not trained or retrained. Most of the cultural practices in India surround left handedness than left footedness.

Right handedness is now thought to be genetically coded. But there is no sufficient information on the inheritance pattern of left handedness. ${ }^{20}$ No genetic basis has been successfully established even in studies on identical twins. Also, the probability of an individual to be left handed with both parents being left handed has been found to be only $35 \%$. Thus, like many other conditions, handedness is also probably determined by a complex gene-environment interaction. Our study showed that $10 \%(n=26)$ of family members of righthanded individuals were left handed but none of the family members of left-handed individuals exhibited left handedness. Pedigree analysis of these families failed to demonstrate any typical Mendelian pattern of inheritance (-Table 4). Thus, our study also points to the fact that both hereditary and external environmental factors play a role in determining left sidedness. Studies have also shown a higher male predisposition to left sidedness. ${ }^{21}$ Left handedness was thought to be historically associated with mental retardation, emotional and behavioral problems, low immunity, low-academic performance and learning disabilities, poor speech, poor control of hands, epilepsy, sudden cardiac death, migraine, inflammatory bowel disease, and a shorter life span, though most of these postulates lack scientific evidence. But because of these beliefs retraining of dexterity is common in many races. This has resulted in several problems in the child, like stuttering, stammering, and dyslexia all due to a mechanism known as cerebral confusion which happens during retraining where the cerebral dominance gets shifted. ${ }^{6,22}$ Ambidexterity is one possible outcome of retraining. Several studies have proven the association between schizotypy and schizophrenia with mixed handedness and mixed footedness. ${ }^{23}$ This study has shown that positive history of retraining and handedness are statistically significant. Hence, individuals who have been retrained for switching of left handedness and have successfully switched over to right handedness/mixed handedness should be followed-up for adverse events like stuttering, stammering, dyslexia, personality problems, and psychiatric manifestations which are probable side effects of the induced cerebral confusion.

\section{Conclusions}

Lateral preferences and handedness had only weak association, but lateral preferences and footedness had statistically significant association. The predictive capacity of HC and AF is more reliable for footedness than handedness probably because of absence of retraining. Since retraining of footedness is seen lesser in society, it should be considered a more superior factor for assessing cerebral dominance. 
Hence, from this study, it can be inferred that RHC and LAF are better determinants of right-side dominance that is left-hemispheric dominance. The reason for different conclusions by different authors indicates that there are still grey areas in the study of sidedness, as it is determined by genetic factors, environmental factors, and several intrauterine causes like testosterone. Association between cerebral dominance and sidedness needs further evaluation as measures of cerebral dominance are clinically important in the diagnosis, treatment and prognosis of strokes and aphasias, speech problems, and dyslexia. As the current study is an observational study, the results need not be conclusive. Effects of age on laterality need to be further investigated by repeating the study in varied age groups in future research. Genetic and environmental factors deciding sidedness can also be studied. Further addition of electroencephalography (EEG)/magnetic resonance imaging (MRI)/functional MRI may throw more light into the topic.

\section{Funding}

The study was funded by The Institute Intramural Research Fund.

\section{Authors' Contributions}

A.M.: methodology, data curation, and writing original draft preparation.

S.R.: methodology, writing, and reviewing.

R.D.: conceptualization, writing, reviewing, and editing. The study was approved by institutional research committee and institutional ethics committee.

\section{Conflict of Interest}

None declared.

\section{Acknowledgments}

The authors thank the medical and nursing students for their cooperation and participation in the study. They also thank the Departments of Pharmacology and Forensic Medicine of their institute for providing permission to use their resources and time in the conduct of this research.

\section{References}

1 Hebbal GV, Mysorekar VR. Evaluation of some tasks used for specifying handedness and footedness. Percept Mot Skills 2006;102(1):163-164

2 Elias LJ, Bryden MP, Bulman-Fleming MB. Footedness is a better predictor than is handedness of emotional lateralization. Neuropsychologia 1998;36(1):37-43
3 Elias LJ, Bryden MP. Footedness is a better predictor of language lateralisation than handedness. Laterality 1998;3(1):41-51

4 Ogah I, Stewart E, Treleaven M, Wassersug RJ. Hand clasping, arm folding, and handedness: relationships and strengths of preference. Laterality 2012;17(2):169-179

5 Mohr C, Thut G, Landis T, Brugger P. Arm folding, hand clasping, and Luria's concept of "latent left-handedness". Laterality 2006;11(1):15-32

6 Kushner HI. Retraining the King's left hand. Lancet 2011;377(9782):1998-1999

7 Siebner HR, Limmer C, Peinemann A, et al. Long-term consequences of switching handedness: a positron emission tomography study on handwriting in "converted" left-handers. J Neurosci 2002;22(7):2816-2825

8 Freire-Maia A, de Almeida J. Hand clasping and arm folding among African Negroes. Hum Biol 1966;38(3):175-179

9 Singh NR, Malhotra KC. Hand clasping and arm folding among the Manipuris (India). Hum Hered 1971;21(2):203-207

10 Sainburg RL, Duff SV. Does motor lateralization have implications for stroke rehabilitation? J Rehabil Res Dev 2006;43(3):311-322

11 Steenhuis RE, Bryden MP, Schwartz M, Lawson S. Reliability of hand preference items and factors. J Clin Exp Neuropsychol 1990;12(6):921-930

12 Yang N, Waddington G, Adams R, Han J. Translation, cultural adaption, and test-retest reliability of Chinese versions of the Edinburgh handedness inventory and waterloo footedness questionnaire. Laterality 2018;23(3):255-273

13 Eligar RC. The laterality of handedness, foot preference and foot overlapping. J Clin Diagn Res 2011;5:421-424

14 McManus IC, Mascie-Taylor CG. Hand-clasping and arm-folding: a review and a genetic model. Ann Hum Biol 1979;6(6):527-558

15 Reiss $M$. The genetics of hand-clasping-a review and a familial study. Ann Hum Biol 1999;26(1):39-48

16 Reiss M. Leg-crossing: incidence and inheritance. Neuropsychologia 1994;32(6):747-750

17 Watson GS, Pusakulich RL, Ward JP, Hermann B. Handedness, footedness, and language laterality: evidence from Wada testing. Laterality 1998;3(4):323-330

18 Day LB, MacNeilage PF. Postural asymmetries and language lateralization in humans (Homo sapiens). J Comp Psychol 1996;110(1):88-96

19 Strauss E, Wada J. Lateral preferences and cerebral speech dominance. Cortex 1983;19(2):165-177

20 McManus IC. The inheritance of left-handedness. Ciba Found Symp 1991;162:251-267

21 Plato CC, Fox KM, Garruto RM. Measures of lateral functional dominance: hand dominance. Hum Biol 1984;56(2):259-275

22 Kushner HI. Retraining left-handers and the aetiology of stuttering: the rise and fall of an intriguing theory. Laterality 2012;17(6):673-693

23 Tran US, Stieger S, Voracek M. Mixed-footedness is a more relevant predictor of schizotypy than mixed-handedness. Psychiatry Res 2015;225(3):446-451 